\title{
Caracterización reológica de la disolución mucilaginosa de cadillo Triumfetta aff Molissima HBK, empleado en la clarificacion de jugos de caña
}

\author{
${ }^{1}$ Teresa del Socorro Blanco Tirado, ${ }^{2}$ Lourdes Margarita Zumalacárregui de Cárdenas \\ ${ }^{1}$ Instituto Universitario de la Paz, Avenida Santander No. 10-22, Barrancabermeja, \\ Colombia.E-mail: sanico@latinmail.com \\ ${ }^{2}$ Instituto Superior Politécnico "José A. Echeverría", Calle 127, s/n CP 19390 \\ Marianao, Ciudad de La Habana, Cuba.E-mail: lourdes@quimica.ispjae.edu.cu
}

\begin{abstract}
Resumen
El comportamiento reológico del mucílago obtenido de las cortezas de cadillo fue estudiado en función de la concentración a la cual se preparó la disolución y de las temperaturas a las cuales se encuentran los jugos de caña en el momento de la aplicación de los aglutinantes. Para la evaluación de las disoluciones se empleó un viscosímetro rotacional Brookfield DV II. Se usó el modelo de la ley de la potencia para describir el comportamiento de los valores de la velocidad de cizalla y del esfuerzo cortante, además para determinar el índice de consistencia (K) y el índice de flujo (n). La disolución mucilaginosa de cadillo preparado en jugo de caña exhibió un comportamiento pseudoplástico con $\mathrm{n}<1$. Los resultados obtenidos en el estudio permiten determinar, con una confiabilidad del 95\%, que la disolución mucilaginosa de cadillo pierde sus características aglutinantes en la medida en que se somete a la acción de la temperatura, siendo más susceptibles las disoluciones preparadas a concentraciones bajas. Se obtuvieron modelos matemáticos que describen el comportamiento reológico y la viscosidad aparente de la disolución mucilaginosa de cadillo en función de la concentración y de la temperatura.
\end{abstract}

Palabras claves: Caracterización reológica, pseudoplásticos, modelos matemáticos, mucílagos, cadillo.

\begin{abstract}
Rheological behavior of mucilage obtained from two barks of cadillo (Triumfetta aff. molissima. HBK) was studied as a function of the concentration at wich dissolution was prepared and of temperatures of cane juices in the moment of application of aglutinante. In order two test the dissolutions a Brookfield DV II rotationatal viscosimeter was used. The power law model was used to describe shear rate speed values and shear stress; also the consistency index $(K)$ and flow behavior index ( $n)$ where determined. Mucilage dissolution showed a pseudoplastic behavior, $n<1$. Results read to determine with high reliability that cadillo mucilage lacks its aglutinant properties as it is submited to the effects temperature. Mathematical models describing rheological behavior and aparent viscosity of the mucilage were obtained as a function of the concentration and temperature.
\end{abstract}

Keywords: Rheological behavior, Pseudoplastics, Mathematical Models, Mucilage, Cadillo. 


\section{Introducción}

En el proceso de producción de panela, los productores colombianos utilizan cortezas de balso (Heliocarpus popayanensis), guásimo (Guazuma ulmifolia. Lamarck) y cadillo (Triumfetta aff. Molissima. HBK) que al ser desprendidas y maceradas desprenden una baba mucilaginosa que facilita la labor de limpieza de los jugos de caña al actuar como aglutinantes de los sólidos en suspensión (CORPOICA-CIMPA, 1999).

Los trabajos de investigación sobre limpieza de jugos de caña mediante la adición de mucílagos han estudiado variables tales como temperatura del jugo al momento de aplicación del aglutinante; concentraciones de aglutinante adecuadas con relación al volumen total del jugo y velocidad de calentamiento de los jugos en presencia del aglutinante, entre otras, y su relación con el poder clarificador de los aglutinantes, medido en términos de la cantidad de impurezas presentes en el producto final panela (Ruiz,1998; Garcia, 2001). Hasta el momento no hay estudios que permitan discutir el comportamiento del aglutinante frente a las variables temperatura y concentración empleadas durante la clarificación de los jugos de caña.

El presente trabajo busca contribuir al conocimiento de la solución mucilaginosa preparada a partir de la corteza del cadillo determinando su comportamiento reológico y la dependencia de los parámetros reológicos índice de consistencia $(\mathrm{K})$ e índice de flujo (n) con la temperatura y la concentración, con el fin de hacer más eficiente el uso del cadillo y avanzar en la industrialización del aglutinante. En este sentido se determinaron modelos matemáticos que describen el comportamiento reológico del mucílago con sus parámetros $\mathrm{K}$ y n en función de las variables de estudio concentración y temperatura.

\section{Proceso de limpieza de jugos de caña en Colombia}

La clarificación mediante cortezas se suele realizar de dos maneras diferentes:

- Utilizando la corteza macerada para formar manojos o escobillas que sirven para "barrer" los jugos cuando alcanzan temperaturas superiores a los $50^{\circ} \mathrm{C}$. La cantidad de corteza a emplear depende de la capacidad del trapiche y de la zona. En la Hoya del Río Suárez se emplean escobillas de $7 \mathrm{~kg}$ de corteza por cada 500 litros de jugo, lo que equivale a una concentración del 1,4\% p/v (Garcia, 2001).

- Añadiendo al jugo una solución clarificante que se prepara sumergiendo la corteza macerada en agua o en jugo de caña crudo, hasta obtener un líquido viscoso. La cantidad de solución mucilaginosa a emplear depende de la concentración de la misma, de la variedad de caña, de la calidad de los jugos y de las condiciones climáticas de la zona. En la Hoya del Río Suárez se emplean entre 15 y 30 litros de solución por cada 500 litros de jugo. Esto es, a una concentración del 3 al 6\% v/v. Cuando los jugos alcanzan temperaturas superiores a los $50^{\circ} \mathrm{C}$ se agregan $3 / 4$ partes de la preparación mucilaginosa y cuando el jugo está entre los 75 y $82^{\circ} \mathrm{C}$ se retira manualmente la cachaza negra conformada por las impurezas que flotan. Una vez retirada la cachaza negra se adiciona el $1 / 4$ de preparación restante y antes que los jugos alcancen la ebullición se remueve la segunda capa de impurezas llamada cachaza blanca. 


\section{Mucílagos vegetales}

Los mucílagos son productos orgánicos de origen vegetal, de peso molecular elevado, superior a $200.000 \mathrm{~g} / \mathrm{gmol}$, cuya estructura molecular completa es desconocida. Están compuestos por polisacáridos celulósicos que contienen el mismo número de azúcares que las gomas y las pectinas, diferenciándose de éstas sólo en las propiedades físicas: mientras que las gomas se hinchan en el agua para dar dispersiones coloidales gruesas y las pectinas se gelifican, los mucílagos producen soluciones viscosas que presentan actividad óptica y pueden ser hidrolizados y fermentados (CIMPA, 1992).

Entre las numerosas macromoléculas de origen natural, un cierto número poseen la propiedad de dispersarse fácilmente en el agua para dar lugar a un aumento bastante consecuente de la viscosidad y a veces a un efecto gelificante. Los mucílagos vegetales hacen parte de estas macromoléculas que se encuentran en las células que conforman tallos, hojas, raíces y frutos de una amplia variedad de especies vegetales (LindenLorient, 1996). Estas disoluciones hidrocoloidales pueden presentarse en estructuras lineales o ramificadas. Cuando los polímeros son lineales presentan en disolución, alta viscosidad aparente, debido a que al ser hidrófilos, interaccionan con el agua adyacente inmovilizándola. Con ello, las moléculas adquieren mayor volumen, siendo su viscosidad aparentemente elevada en relación con su masa molar y aumentando considerablemente con la concentración. Cuando los polímeros presentan cadenas ramificadas, ocupan menor volumen que los de cadenas lineales y por ello su viscosidad aparente es menor (Muller, 1992). Desde diferentes estudios sobre el proceso de clarificación de los jugos de caña se sostiene que los mucílagos se emplean con fines floculantes, coagulantes y aglutinantes, esto gracias a sus propiedades lipófilas e hidrófilas que conjuntamente, lo que les permite es que al contacto con el agua se polimericen y formen redes que ayudan a retener partículas orgánicas.

\section{Reología de fluidos}

Un fluido es una sustancia que sufre continuas deformaciones cuando se le somete a una fuerza de cizalla. Una fuerza de cizalla es aquella que permite que varias láminas planas colocadas en paralela deslicen unas sobre otras. La fuerza de cizalla produce deformación, es decir, un cambio en la posición relativa de las partes de un cuerpo. Para producir el movimiento de un fluido debe aplicársele una fuerza de cizalla.

La viscosidad es la propiedad que afecta principalmente al comportamiento de un fluido y está relacionada con la resistencia que ejerce el fluido al movimiento. La viscosidad constituye un importante aspecto de la reología, la ciencia de la deformación y del flujo. La viscosidad se determina relacionando el gradiente de velocidad en los fluidos y la fuerza de cizalla que genera el flujo de la forma como se relaciona en la siguiente figura: 


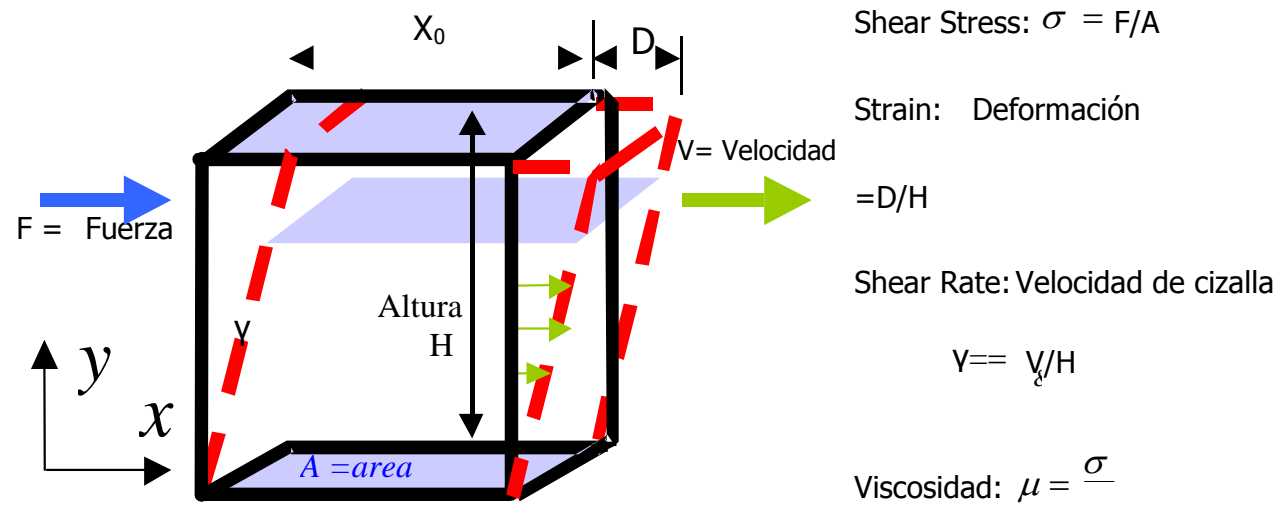

Figura 1. Viscosidad y prueba de cizallamiento

En fluidos Newtonianos el esfuerzo cortante $(\sigma)$ y la velocidad de cizalla $(\Varangle)$ se relacionan directamente y su comportamiento se puede describir empleando el siguiente modelo:

$$
\sigma=-\mu \frac{d v}{d y} \quad \text { Ec. } 1
$$

Esta ecuación es la Ley de la viscosidad de Newton, donde $\mu$ es la viscosidad del fluido. (Doran, 1998). Muchos fluidos biológicos son no newtonianos y no obedecen al comportamiento descrito por la Ec. 1. El esfuerzo cortante $(\sigma)$ es dependiente de la velocidad de cizalla $(\gamma)$ y en varios casos, dependientes del tiempo (Rao, 1977). Allen, Robinson, Manohar citados por Oliveros afirman que además, el comportamiento reológico de los materiales biológicos también está influenciado por la concentración de biomasa y que por lo tanto, se requieren modelos más complejos para su estudio (Oliveros,1996). Los modelos de Herschel-Bulkley y de Casson se usan ampliamente para ajustar los datos de $\sigma$ y $\gamma$ (Rao 1977)

$$
\begin{array}{lll}
\text { Modelo Herschel-Bulkley: } & \sigma=\sigma_{y}+K \dot{\gamma}^{n} & \text { Ec. } 2 \\
\text { Modelo de Casson: } & \sigma_{y}=\sigma_{y}^{1 / 2}+K_{c} \dot{\gamma}^{1 / 2} & \text { Ec. } 3
\end{array}
$$

De donde: $\sigma_{y}=$ Esfuerzo inicial; $\mathrm{K}=$ Indice de consistencia; $\mathrm{n}=$ Indice de flujo; $\mathrm{K}_{\mathrm{c}}=$

Constante de Casson. En los fluidos que exhiben un esfuerzo inicial bajo se usa el modelo de la Ley de la Potencia (OLIVEROS,1996). Este modelo puede ser considerado un caso especial del modelo de Herschel-Bulkley.

Modelo de la Ley de la Potencia: $\sigma=K \dot{\gamma}^{n} \quad$ Ec. 4

Basados en la magnitud de $\mathrm{n}$ y de $\sigma_{y}$ los fluidos se pueden agrupar como: Newtonianos $\left(\mathrm{n}=0, \sigma_{y}=0\right)$; Bingham $\left(\mathrm{n}=0, \sigma_{y}>0\right)$; pseudoplásticos $\left(0<\mathrm{n}<1, \sigma_{y}=0\right)$; Binghampseudoplásticos $\left(0<\mathrm{n}<1, \sigma_{y}>0\right)$. La viscosidad aparente $\left(\eta_{a}\right)$ reportada para fluidos a 
los que sus parámetros reológicos $(\sigma)$ y $(\dot{\gamma})$ se ajustan a la Ley de la Potencia esta dada por:

$$
\eta_{a}=\kappa \dot{\gamma}^{n-1} \quad \text { Ec. } 5
$$

A diferencia de los fluidos Newtonianos, en los fluidos no Newtonianos la viscosidad tiende a disminuir en la medida en que se incrementan los valores del gradiente de cizallamiento. Según RAO, citado por Oliveros, los fluidos compuestos de substancias de alto peso molecular tales como los mucílagos exhiben un comportamiento no Newtoniano.

(a) $1.3 \%$

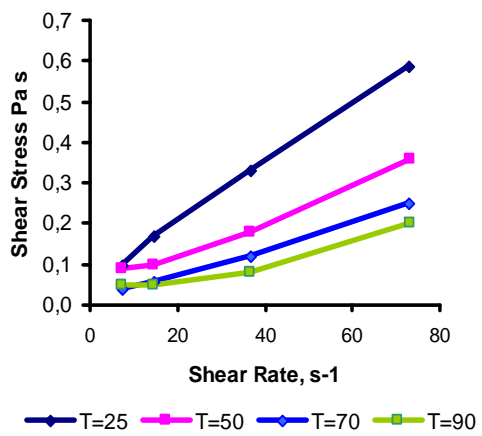

(b) $1.4 \%$

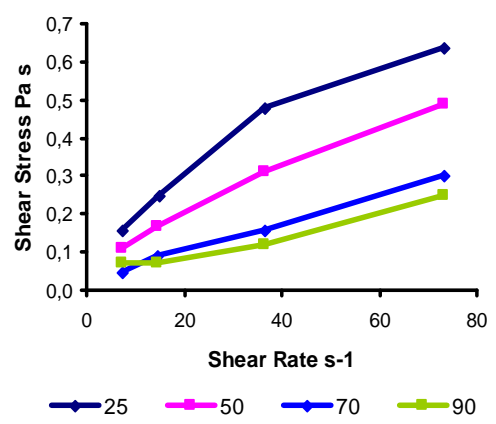

(c) $1,5 \%$

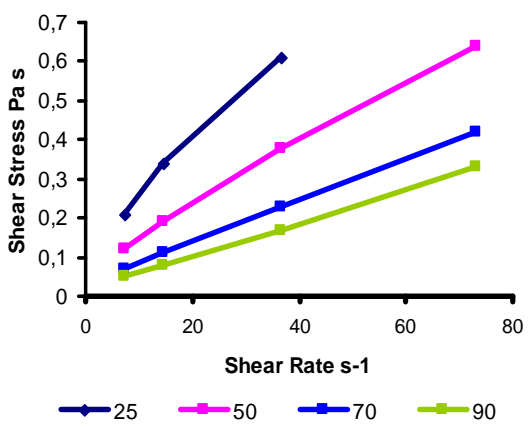

Figura 2. Reogramas para los tratamientos $\mathrm{C}^{*} \mathrm{~T}$ de la disolución mucilaginosa de Cadillo. Fuente: La Autora.

\section{Materiales y métodos}

Para el estudio se empleó cortezas maceradas de cadillo disueltas en jugo de caña, variedad RD 7511, siguiendo la metodología sugerida por Acero y Pacheco (2001a) para la obtención del mucílago. El jugo mostró un pH 5 y $16^{\circ} \mathrm{Bx}$ en el momento de la mezcla. Las soluciones se prepararon a tres concentraciones $(1.3,1.4$ y $1.5 \mathrm{p} / \mathrm{v})$, teniendo en cuenta las concentraciones reportadas por la literatura al respecto. De igual forma, se estudiaron a cuatro niveles de temperatura $\left(25,50,70\right.$ y $\left.90^{\circ} \mathrm{C}\right)$ para formar 12 tratamientos, evaluados con tres replicaciones. Para la caracterización de los parámetros 
reológicos se empleó un viscosímetro rotacional Brookfield DVII con el adaptador LV ULA00. Las lecturas se hicieron en orden aleatorio, se tomaron tres valores para un mismo tratamiento con una diferencia de tiempo entre ellos de 30 segundos. Se hicieron lecturas a 60, 30, 12 y $6 \mathrm{rpm}$ y se obtuvieron valores para esfuerzo cortante, velocidad de cizalla y viscosidad. Se empleó el método de regresión lineal múltiple para determinar el efecto de la concentración (C) y de la temperatura (T) sobre el índice de consistencia (K) y el índice de flujo (n).

\section{Resultados y Discusión}

\section{Caracterización reológica}

La figura 2 muestra los reogramas construidos a partir de los datos de esfuerzo cortante $(\sigma)$ Vs la velocidad de cizalla $(\dot{\gamma})$ para cada uno de los tratamientos $\mathrm{C}^{*} \mathrm{~T}$ del estudio. Con base en estos reogramas y según lo reportado por Oliveros,1996; Zumalacarregui, 2001; Steffe, 1996, se puede afirmar que la disolución mucilaginosa de cadillo es un fluido pseudoplástico con $\sigma_{y}=0$ y $0<$ n $<1$. (Ver Tabla 1$)$.

(a) $1.3 \%$

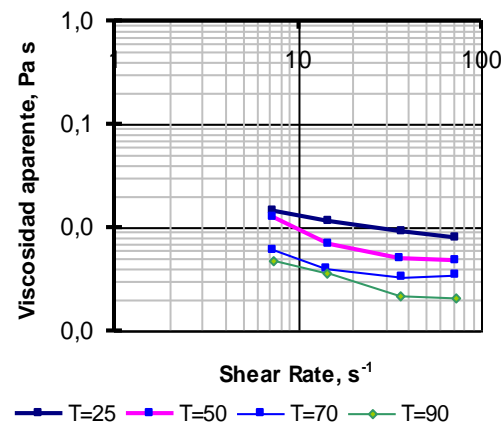

(b) $1.4 \%$

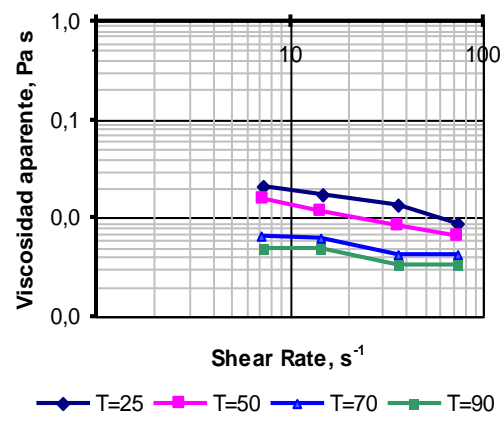

(c) $1.5 \%$

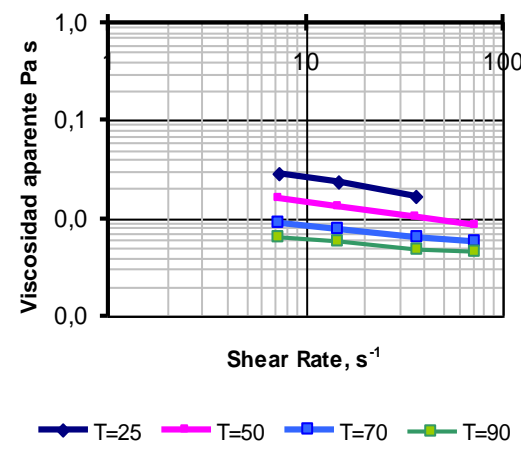

Figura 3. Relación entre la Viscosidad aparente y la velocidad de cizalla para los tratamientos $\mathrm{C}^{*} \mathrm{~T}$ de la disolución mucilaginosa de Cadillo. Fuente: La Autora. 
En la Figura 3 se graficaron los valores de viscosidad aparente $\left(\eta_{a}\right)$, calculados según la Ec 5, Vs la velocidad de cizalla $(\dot{\gamma})$ a escala log-log. En esta figura se puede apreciar que, para todos los tratamienos $\mathrm{C}^{*} \mathrm{~T}$, la viscosidad tiende a disminuir en la medida en que se incrementan los valores de la velocidad de cizalla, tendencia característica de los fluidos pseudoplásticos. (Barnes, H. A.; Mutton, J. F., 1997).

\section{Análisis estadístico de los resultados}

En la Tabla 1 se muestran los valores de $\mathrm{K}$ y n para cada tratamiento $\mathrm{C} * \mathrm{~T}$. Los valores de índice de consistencia (K) e índice de flujo (n) se obtuvieron correlacionando los valores de $(\sigma)$ y $(\dot{\gamma})$ según cada tratamiento $\mathrm{C}^{*} \mathrm{~T}$. El modelo que se ajustó al comportamiento de estos datos con $\mathrm{r}^{2}>0.96$ fue el de la ley de la potencia representado por la Ec. 4. En general, para todas las concentraciones los valores de K decrecen en la medida en que se incrementa la temperatura.

Tabla 1. Valores de $\mathrm{K}$ y n según tratamiento $\mathrm{C} * \mathrm{~T}, \mathrm{C}=$ concentración $(1,3 ; 1,4 ; 1,5 \%$ $\mathrm{p} / \mathrm{v}) \mathrm{T}=$ temperatura $\left(25,50,70,90^{\circ} \mathrm{C}\right)$

Fuente: La Autora

\begin{tabular}{ccc}
\hline Tratamiento $\mathrm{C}^{* \mathrm{~T}}$ & $\begin{array}{c}\text { Índice de } \\
\text { consistencia }(\mathrm{K})\end{array}$ & $\begin{array}{c}\text { Índice de } \\
\text { flujo }(\mathrm{n})\end{array}$ \\
\hline $\mathrm{C}_{1} \mathrm{~T}_{1}\left(1,3 \% \mathrm{p} / \mathrm{v}^{*} 25^{\circ} \mathrm{C}\right)$ & 0.02 & 0.79 \\
$\mathrm{C}_{1} \mathrm{~T}_{2}$ & 0.01 & 0.76 \\
$\mathrm{C}_{1} \mathrm{~T}_{3}$ & 0.005 & 0.91 \\
$\mathrm{C}_{1} \mathrm{~T}_{4}$ & 0.004 & 0.87 \\
$\mathrm{C}_{2} \mathrm{~T}_{1}$ & 0.05 & 0.57 \\
$\mathrm{C}_{2} \mathrm{~T}_{2}$ & 0.029 & 0.65 \\
$\mathrm{C}_{2} \mathrm{~T}_{3}$ & 0.01 & 0.79 \\
$\mathrm{C}_{2} \mathrm{~T}_{4}$ & 0.01 & 0.74 \\
$\mathrm{C}_{3} \mathrm{~T}_{1}$ & 0.058 & 0.65 \\
$\mathrm{C}_{3} \mathrm{~T}_{2}$ & 0.026 & 0.74 \\
$\mathrm{C}_{3} \mathrm{~T}_{3}$ & 0.012 & 0.82 \\
$\mathrm{C}_{3} \mathrm{~T}_{4}$ & 0.008 & 0.88 \\
\hline
\end{tabular}

Tabla 2. Análisis de regresión múltiple para el índice de flujo (n) y su respectivo ANOVA. Fuente: La Autora.

Multiple Regresion Analysis

\begin{tabular}{lrrrr}
\hline Dependent variable: $n$ & \multicolumn{5}{c}{ T } & p-Value \\
\hline & Estimate & Standar & Statistic & \\
& & error & 0,0040 \\
Concentración & 1,13099 & 0,294911 & 3,83501 & 0,0158 \\
Temperatura & 0,00281887 & 0,000950507 & 2,96565 & 0,0150 \\
Concentración $^{\wedge} 2$ & $-0,0500827$ & 0,203074 & $-2,46624$ & 0,0358 \\
\hline
\end{tabular}


Analysis of Variance

\begin{tabular}{lrrrrr}
\hline Source & Sum of Squares & Df & Mean Square & F-Ratio & p-Value \\
\hline & & & & & \\
Model & 7,06614 & 3 & 2,35538 & 374,77 & 0,0000 \\
Residual & 0,0565644 & 9 & 0,00628494 & & \\
\hline
\end{tabular}

Total $\quad 7,1227 \quad 12$

$R$-squared $=99,2059$ percent

$R$-squared $=($ adjusted for $d . f)=99,$.0294 percent

Standar Error of Est. $=0,0792776$

Mean absolute error $=0,0554511$

El valor de p para la variable dependiente $\mathrm{n}$ es menor que 0.01. Esto significa que se puede afirmar con un nivel de confianza del 99\% que existe una interrelación estadísticamente significativa entre la variable dependiente índice de flujo (n) y las variables independientes concentración y temperatura.

La Tabla 3 muestra el análisis correspondiente a la variable índice de consistencia $(\mathrm{K})$ :

Tabla 3. Análisis de regresión múltiple para la variable índice de consistencia K. Fuente: La Autora.

Multiple Regresion Analysis

Dependent variable: $K$

\begin{tabular}{lrrrr} 
Parameter & Estimate & Estándar & $T$ & $p$-Value \\
\hline & & Error & Statistic & \\
Concentración & 0,0389181 & 0,00505468 & 7,69942 & 0,0000 \\
Temperatura & $-0,000580717$ & 0,000111645 & $-5,20146$ & 0,0004 \\
\hline
\end{tabular}

Analysis of Variance

\begin{tabular}{lrrrrr}
\hline Source & Sum of Squares & Df & Mean Square & F-Ratio & p-Value \\
\hline & & & & & \\
Model & 0,00744543 & 3 & 0,00372271 & 42,08 & 0,0000 \\
Residual & 0,000884571 & 9 & 0,0000884571 & & \\
\hline
\end{tabular}

Total $\quad 0,00833 \quad 12$

$R$-squared $=89,3809$ percent

$R$-squared $=($ adjusted for d.f. $)=88,319$ percent

Standar Error of Est. = 0,00940516

Mean absolute error $=0,00737846$ 
El valor de p para la variable dependiente $\mathrm{K}$ es menor que 0.01. Esto significa que se puede afirmar con un nivel de confianza del $99 \%$ que existe una interrelación estadísticamente significativa entre la variable dependiente índice de consistencia (K) y las variables independientes concentración y temperatura.

Modelos matemáticos que describen el comportamiento reológico de la disolución mucilaginosa de cadillo con los parámetros reológicos $\mathrm{K}$ y n en función de las variables en estudio concentración y temperatura:

$$
\begin{aligned}
& \left.\sigma=[-1.597+2.161 C-1.8632 E-05 T] \dot{\gamma}^{\left[23.804-32.5 C+11.311 C^{2}-0.0014 T\right.}\right] \\
& \eta_{a}=[-1.597+2.161 C-1.8632 E-05 T] \dot{\gamma}^{\left[23.804-32.5 C+11.311 C^{2}-0.0014 T\right]^{-1}}
\end{aligned}
$$

Estos modelos matemáticos sirven para predecir el comportamiento de la viscosidad del mucílago durante la preparación de la panela. Aquí es importante tener en cuenta que el efecto clarificador del mucílago está relacionado directamente con la viscosidad del mismo

\section{Conclusiones}

La disolución mucilaginosa de cadillo preparada en jugo de caña se puede clasificar como un fluido pseudoplástico debido a que el comportamiento de sus parámetros reológicos $(\sigma)$ y $(\dot{\gamma})$ se ajustaron con un $\mathrm{r}^{2}>0.96$ al modelo de la ley de la potencia y los valores del índice de flujo (n) para todos los tratamientos evaluados fue menor de 1.

El comportamiento de la viscosidad aparente $\left(\eta_{a}\right)$ Vs gradiente de cizalla $(\curlyvee)$ para los valores obtenidos en el estudio, se ajusta a lo reportado por la literatura en lo que respecta a la tendencia en el comportamiento de estos dos parámetros en los fluidos pseudoplásticos: el valor de la viscosidad aparente disminuye en la medida en que se hacen incrementos paulatinos en la velocidad de cizalla, pero este descenso tiende a estabilizarse cuando se superan niveles altos de cizalla.

La temperatura y la concentración tienen un efecto significativo sobre el índice de consistencia $\mathrm{K}$ y sobre el índice de flujo $\mathrm{n}$, y teniendo en cuenta que el comportamiento del mucílago se ajusta al modelo de la ley de la potencia, se puede afirmar que estas dos variables actúan directamente sobre la viscosidad del mucílago.

En la práctica, y a la luz de los resultados sobre la evaluación reológica de la disolución mucilaginosa de cadillo se puede inferir, con un $99 \%$ de confianza, que no es conveniente someter los mucílagos a temperaturas de procesamiento altas.

\section{Recomendaciones}

Hacer nuevas experimentaciones tomando las muestras a evaluar directamente de la paila donde se cocinan los jugos y en el momento de aplicación de los mucílagos.

Evaluar el efecto de las variedades de cañas en el comportamiento reológico de la disolución mucilaginosa de cadillo. 
Evaluar el efecto del tiempo que duran en contacto con los jugos las escobillas fabricadas con las cortezas maceradas de cadillo sobre el comportamiento reológico de su disolución mucilaginosa.

Evaluar reológicamente las disoluciones variando el grado de madurez de las cortezas empleadas para la extracción del mucílago.

Hacer nuevos experimentos donde se amplié el tiempo de disgregación del mucílago en la etapa inicial de preparación.

Hacer nuevas evaluaciones reológicas donde se amplíen los rangos de concentración del mucílago, y se empleen otras especies vegetales.

Evaluar simultáneamente al comportamiento reológico el efecto clarificador de los mucílagos frente a las variables concentración y temperatura.

\section{Bibliografía}

[D] Acero V, Osman L y Pacheco R, Guerley H. (2001a) Elaboración de la fichas técnicas de las disoluciones mucilaginosas del balso Heliocapus popayanensis, cadillo Triumpetta aff. Molissima H.K.B. y guásimo Guazuma ulmifolia. Lamarck. Tunja, 2001., Proyecto de grado (Químicos de Alimentos). Universidad Pedagógica y Tecnológica de Colombia. Facultad de Ciencias. Escuela de Química de Alimentos.

(2001b). Estandarización de la metodología para la separación y purificación del mucílago de Balso Heliocarpus popayanensis, HBK. Trabajo de pasantía. CORPOICA-CIMPA. 2001.

미 Barnes, H.A; Mutton, J. Viscosity. En: Handbook of Food Engineering, Elsevier science: 1997. 11-35 p.

ㅁd Doran, Pauline. Principios de ingeniería de los bioprocesos. Editorial Acribia, España:1998.

[D] Garcia, Gerardo. Mejoramiento de las condiciones de uso del aglutinante natural guásimo Guazuma ulmifolia. Lamarck. y cadillo Triumpetta aff. Molissima H.K.B en el proceso de elaboración de panela para pequeños y medianos productores. Proyecto de grado (Ingeniero Agroindustrial) Instituto Universitario de la Paz. Barrancabermeja. 2001.

[D] Oliveros, Carlos E. Rheological characterization of coffee mucilage. En: Journal of food process engineering, 19, 331-342 (1996).

[Dd Rao, M. A. Rheology of liquid foods. Journal of texture studies.. pg 135-167.

[D] Steffe, J. Rheological Methods in Food Process Engineering. $2^{\mathrm{a}}$ Edición. EEUU: 1996.

[D] Zumalacarregui, Lourdes. Módulo de Reología UNAD, 2001. 


\title{
Estudio comparativo de arcillas decolorantes utilizadas industrialmente en la operación de blanqueo de aceites de palma
}

\author{
${ }^{1}$ Javier Mauricio Rodríguez Mejía, ${ }^{2}$ Jesús Alfonso Torres Ortega \\ ${ }^{1}$ Escuela de Postgrados, Universidad Nacional Abierta y a Distancia, Calle 45 No. 53 - \\ 39 Bogotá, Colombia, Tel.: +57-1-34760088 Fax: 3472556, E-mail: \\ javier.rodriguez@unad.edu.co, \\ ${ }^{2}$ Departamento de Ingenieria Química, Universidad Nacional de Colombia, Carrera 30 \\ No. 45-03 Bogotá, Colombia, Tel.: +57-1-3165000 Ext.: 14303, Fax: 14301, E- \\ mail: jatorresor@unal.edu.co.
}

\section{Resumen}

El blanqueo de aceites de palma es una operación importante en la refinación en la que suele presentarse inconvenientes, a pesar de que las empresas procesadoras han desarrollado técnicas para controlar la calidad del aceite crudo y hacer más fácil la operación de blanqueo, ésta se considera inestable debido a las variables que se manejan. Durante el blanqueo se retira del aceite trazas de jabones, metales, fosfolípidos y sustancias que aportan color, mediante la acción de arcillas blanqueadoras, las cuales poseen poros y gran área superficial, donde los contaminantes son absorbidos por efecto físico, entregando pureza y calidad al aceite procesado. En el mercado nacional se encuentran varias marcas de arcillas, por esta razón surgió el interés por determinar que arcilla blanqueadora presenta mejores resultados en rendimiento, remoción de contaminantes y mejores propiedades al aceite refinado. Con la información analizada de la experimentación, se evaluó la incidencia de cada variable y se hallaron las mejores condiciones de operación acorde a la información teórica y los resultados experimentales que son presentados en este artículo. En Colombia se encuentran en gran proporción las marcas Tonsil (Sud Chemie) con el 35\%, Pure Flow (Oil Dri) con el 50\% y Filtrol (Engelhard) con el 15\% del mercado. Otras marcas no se encuentran debido a su falta de competitividad en precio.

Palabras claves: Blanqueamiento, aceite de palma, arcillas, refinación.

\begin{abstract}
The palm oil bleaching is an important operation in the refinement in which usually appears inconvenient, although the company's processors have developed technical to control the quality of the crude oil and to conduct the operation easier of bleaching, is considered unstable due to the variables that are handled. During the bleaching is retires of the oil soap plans, metals, phospholipids and substances that contribute colour, by means of the clay action launderers, who have pores and great superficial area, where the polluting agents are absorbed by physical effect, giving to purity and quality to the processed oil. In the national market are several clay marks; therefore arose the interest to determine that clay launderer displays better results in yield, removal of polluting agents and good properties to the refined oil. With the analyzed information of the experimentation, the incidences of each variable was evaluated and were the best conditions of agreed operation to the theoretical information and the experimental results that are presented in this article. In Colombia the Tonsil marks are in great proportion (Sud Chemie) with 35\%, Pure Flow (Oil Dri) with 50\% and Filtrol (Engelhard) with $15 \%$ of the market. Other marks are not due to their lack of competitiveness in price.
\end{abstract}

Key words: Bleaching, palm oil, clays, refinement. 


\section{Introducción}

El aceite de palma crudo de buena calidad se elabora en el campo, no en la fábrica. A medida que la calidad del aceite de palma crudo se deteriora, la eficacia de la totalidad del proceso de la refinería disminuirá independientemente de las operaciones de refinación y del equipo. La calidad final de aceite depende de la eficiencia en la remoción de las impurezas. Cada etapa del proceso de refinamiento esta enfocada en mejorar la calidad del aceite, el objetivo esencial de las operaciones combinadas es producir productos finales de aceite de alta calidad con respecto al sabor, estabilidad oxidativa y características de rendimiento. El blanqueo por definición, es la interacción física y química de un absorbente con un aceite o grasa para mejorar la calidad del producto final. El objetivo de la operación de blanqueo es remover contaminantes presentes en el aceite que son perjudiciales para la calidad del aceite final y no se eliminan mediante los procesos anteriores al blanqueo como jabones, trazas metálicas, fosfolípidos, clorofila y productos de oxidación. Las arcillas blanqueadoras poseen un color amarillo verdoso o azul grisáceo, el peso de un litro de tierra activada varia entre 0.7 y 1.2 kilos y su peso especifico oscila entre $1.8-2.3$. En atmósfera seca el polvo presenta buena estabilidad y no pierde actividad aun después de un almacenamiento prologando. Las tierras naturales son completamente inertes con los triglicéridos y no modifican las constantes químicas del aceite. Las arcillas ácido activadas originan un ligero aumento en la acidez del aceite. Estas tierras comunican al aceite un olor como “terroso" el cual se elimina en la desodorización subsiguiente. Las tierras que presentan reacción ácida pueden llegar a tener un mayor poder decolorante. Las tierras naturales tienden a absorber menor cantidad de aceite en la operación de filtrado $(18-35 \%)$ en comparación frente a las ácidas (50\%). La velocidad de filtración es también menor con las arcillas naturales. Estas características deben tenerse en cuenta para valorar la influencia de las tierras sobre el costo de operación.

\section{Marco Teórico}

\section{El aceite de palma}

La producción mundial actual de aceite de palma alcanza 9.000.000 MT , la planta Elaeis Guineensis fue introducida desde el África occidental hacia Asia oriental en 1948 por razones ornamentales, desde entonces su estudio comenzó, se identifica que cerca del $56 \%$ del contenido de la pulpa es aceite, principalmente los ácidos grasos que contiene con ácido palmítico y ácido esteárico. El punto de fusión esta entre $35-38{ }^{\circ} \mathrm{C}$. Su composición química varía dependiendo de la región donde sea cultivada.

El fruto de palma contiene una enzima lipofílica en su cáscara, la cual si no es inhibida rápidamente comienza a favorecer la formación de ácidos grasos libres, la inhibición de la enzima se logra mediante cocimiento de los frutos a temperatura de $55{ }^{\circ} \mathrm{C}$. El aceite de palma crudo contiene en promedio $2.5 \%$ de ácidos grasos libres (AG.L.), con humedad media de $0.15 \%$, con esta humedad, la hidrolización y oxidación de compuestos no se llevan a cabo. Es común la practica de dosificar al aceite crudo antioxidante del tipo TBHQ (110 ppm) mezclado con ácido cítrico (365 ppm) para evitar la oxidación, de esta manera también se asegura en parte la obtención de un aceite con excelente color así como la reducción de consumo de arcilla blanqueadora ya que 
se minimiza la acción de sustancias pro oxidantes. Normalmente los aceites de palma crudo contienen alrededor de 9 ppm de Fe y $0.1 \mathrm{ppm}$ de $\mathrm{Cu}$.

Tabla 1. Composición Aceite de Palma

\begin{tabular}{|l|l|l|l|l|}
\hline $\begin{array}{c}\text { Ácido } \\
\text { Graso }\end{array}$ & $\begin{array}{c}\text { Africana } \\
(\boldsymbol{\%})\end{array}$ & $\begin{array}{c}\text { América Latina } \\
(\boldsymbol{\%})\end{array}$ & Malaya $(\boldsymbol{\%})$ & $\begin{array}{c}\text { Stardar Codex A. } \\
(\boldsymbol{\%})\end{array}$ \\
\hline C12:0 & - & Traza & Traza & $<1.2$ \\
\hline C14:0 & 1 & 1 & 1 & $0.5-5.9$ \\
\hline C16:0 & 40 & 44 & 46 & $32-59$ \\
\hline C16:1 & 0.5 & Traza & Traza & $<0.6$ \\
\hline C18:0 & 5 & 5 & 4 & $1.5-8.0$ \\
\hline C18:1 & 40.5 & 40 & 39 & $27-52$ \\
\hline C18:2 & 12 & 10 & 10 & $5.0-14$ \\
\hline C18:3 & 0.5 & - & - & $<1.5$ \\
\hline C20:0 & 0 & 0.5 & Traza & $<1.0$ \\
\hline
\end{tabular}

Fuente: Patterson H.B., Blaeching and Purifying Fats ans Oils Pág. 126

El color del aceite crudo es rojo oscuro y se debe al alto contenido de carotenos (superiores a $2000 \mathrm{ppm}$ ó $0.2 \%$ ). El aceite crudo no se debe someter a altas temperaturas ya que los carotenos pueden oxidarse y si hay presencia de A.G.L. se dará origen a la fijación de color café oscuro el cual es muy difícil de remover. Actualmente para determinar la calidad de un aceite crudo éste se somete a una prueba denominada DOBI (Deterioration Of Bleach Ability Index). Los fosfátidos en el aceite crudo normalmente están en cantidades inferiores al $0.1 \%$

\section{Refinamiento del aceite de palma}

La calidad final de aceite depende de la eficiencia en la remoción de las impurezas. Cada etapa del proceso de refinamiento esta enfocada en mejorar la calidad del aceite (ver tabla 2), el objetivo esencial de las operaciones combinadas es producir productos finales de aceite de alta calidad con respecto al sabor, estabilidad oxidativa y características de rendimiento.

Tabla 2. Impurezas a Retirar en el refinamiento del Aceite de Palma.

\begin{tabular}{|c|c|}
\hline Proceso & Impureza \\
\hline Desgomado & Sólidos Filtrables. Fosfolípidos Hidratables. \\
\hline Refinación & A.G.L., Fosfolípidos, Metales, Pigmentos. \\
\hline Blanqueo & Productos de Oxidación, Fosfolípidos, Pigmentos, \\
& Metales, Jabones. \\
\hline Fraccionamiento & Triglicéridos Sólidos, Ceras. \\
\hline Desodorización & $\begin{array}{c}\text { Vitaminas, Acidos Grasos, Productos de Oxidación, } \\
\text { Pigmentos, Resinas, Elementos Volátiles. }\end{array}$ \\
\hline
\end{tabular}

Fuente: Oil Dri Corp. of America 


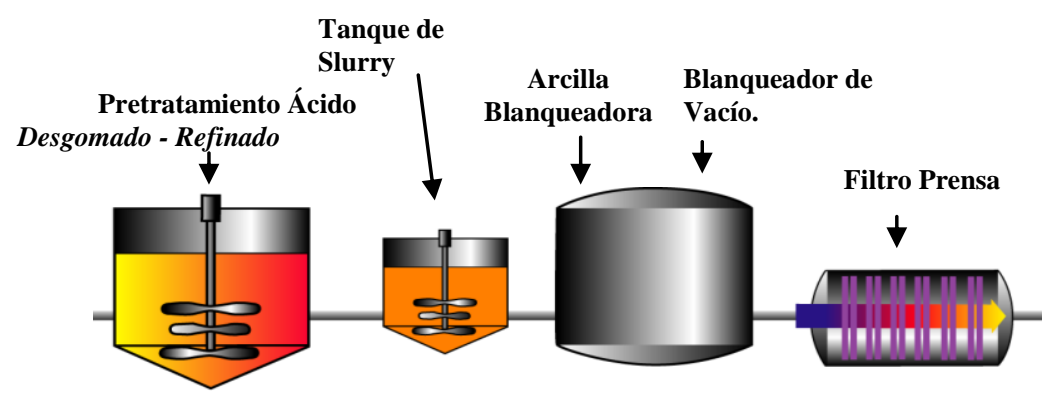

(a)

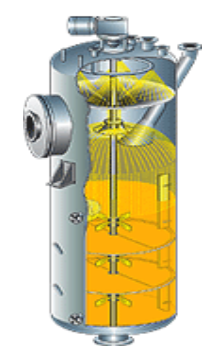

(b)

Figura 1. (a)Proceso refinación física del aceite de palma. (b) Blanqueador Continúo.

\section{Blanqueo de aceite de palma}

Con este proceso se reduce el contenido de carotenoides en el aceite por absorción de tierras de blanqueo hasta un punto en donde el color no exceda a 20 en la escala del Rojo medido por el colorímetro de Lovinbond en celdas de 5 1/2 pulgadas. Esta operación puede realizarse en el mismo tanque neutralizador. El tanque neutralizador se conecta al vacío (para que no se formen productos de oxidación secundaria) y se somete a agitación.

La mezcla blanqueadora se introduce a través de un tubo que llega hasta la mitad del tanque, para evitar la formación de polvo. Las sustancias blanqueadoras ser dosifican según la calidad y estado del aceite tratado en promedio la dosis oscila entre $0,125-$ $2,00 \%$; esta operación remueve los productos de la oxidación de las grasas y cualquier residuo jabonoso que en otras circunstancias afectaría la posterior etapa de desodorización. La figura 1b muestra la estructura interna de un blanqueador moderno de plantas operadoras en serie.

Adicionalmente en esta fase de proceso se remueven pigmentos y otros componentes menores indeseables, las características generales son:

1- Durante la agitación bajo vacío $(50 \mathrm{~mm} \mathrm{Hg})$, el aceite es secado hasta tener máximo $2 \%$ en peso de agua a una temperatura de $80{ }^{\circ} \mathrm{C}$.

2- La arcilla blanqueadora entra al blanqueador mediante vacio.

3- La temperatura se eleva hasta $90-105^{\circ} \mathrm{C}$

4- La dosis de arcilla en promedio esta entre $0.5-1 \%$ en peso.

5- El tiempo de contacto usual esta entre $20-30$ minutos.

6- Posterior al tiempo de contacto, se filtra a temperatura media de $90{ }^{\circ} \mathrm{C}$.

\section{Arcillas Blanqueadoras}

Químicamente las arcillas blanqueadoras son aluminocisilicatos de calcio y sodio que se encuentran en forma natural. Comercialmente se conocen como tierras Fuller o Montmorillonita. Los depósitos más grandes de estos materiales se encuentran en la zona de Georgia en U.S.A. y en Montmorillon, Francia. Dentro de las firmas 
productoras se encuentran Engelhard, SudChemie, Oil Dri, Fulmont, Clarsil. Todas producen las arcillas ácidas y Oil Dri exclusivamente produce la arcilla natural $(\mathrm{pH}$ neutro). Algunas de las características de los productos adsorbentes se enumeran en la tabla 3 .

La actividad del blanqueo se ve afectada por la selección de la materia prima (mineralogía) y el nivel de activación del adsorbente, incluyendo el nivel de acidez, la cantidad e humedad libre y el tamaño y distribución de las partículas del adsorbente pulverizadas.

La arcilla en bruto entra a la planta de procesamiento con humedad libre de $40-50 \%$. Es necesario bajar este nivel a menos de $20 \%$ para que la arcilla sea eficazmente pulverizada y/o activada posteriormente. Esta arcilla es separada de la tierra inerte y demás impurezas mediante sedimentación posteriormente se seca y se pulveriza hasta darle la finura deseada, luego se procede a la acidificación que consiste en rociar ácido clorhídrico o sulfúrico sobre la tierra para nuevamente ser secada, pulverizada, tamizada y empacada.

Tabla 3. Características de Arcillas Adsorbentes.

\begin{tabular}{|c|c|c|}
\hline \multicolumn{2}{|c|}{ Caracteristicas de adsorbentes } \\
\hline Característica & Arcillas Naturales & $\begin{array}{c}\text { Arcillas Acido- } \\
\text { Activa }\end{array}$ \\
\hline Mineralogía & $\begin{array}{c}\text { Mezcla } \\
\text { Attapulgita/Montmorillonita }\end{array}$ & $220-340$ \\
\hline Area Superficial $\left(\mathrm{m}^{2} / \mathrm{g}\right)$ & $110-160$ & $0.20-0.50$ \\
\hline Volumen de Poro $\left(\mathrm{cm}^{3} / \mathrm{g}\right)$ & $0.35-0.62$ & $0.12-0.30$ \\
\hline $\begin{array}{c}\text { Volumen efectivo de Poro } \\
\left(\mathrm{cm}^{3} / \mathrm{g} .5-20 \mu \mathrm{m}\right)\end{array}$ & $0.20-0.30$ & $38-46$ \\
\hline Densidad $\left(\mathrm{lb} / \mathrm{ft}^{3}\right)$ & $27-33$ & $2.5-7.0$ \\
\hline $\mathrm{pH}$ & $2.4-7.0$ & $10.0-16.0$ \\
\hline Humedad Libre \% & $8.0-15.0$ & $30-60$ \\
\hline Promedio & $30-40$ & \\
\hline Tamaño de Partícula $(\mu \mathrm{m})$ & & \\
\hline
\end{tabular}

Fuente: Sud Chemie México.

Únicamente dos clases de minerales sirven como adsorbentes de arcillas blanqueadoras: La montmorillonita y el integrado cristalino Attapulgita / Montmorillonita natural, que se presentan en las figuras 2 y 3 :

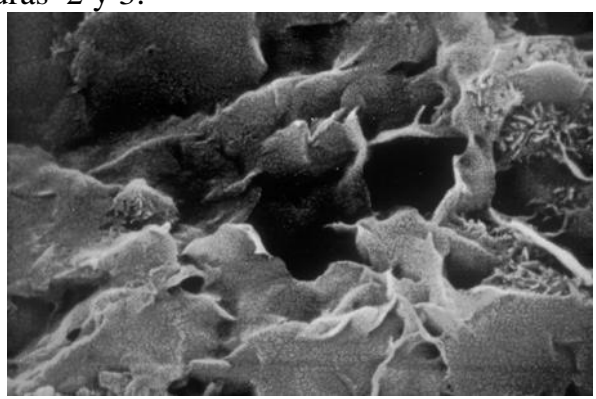

Figura 2. Montmorillonita. Fuente: Oil Dri Corp. of America. 


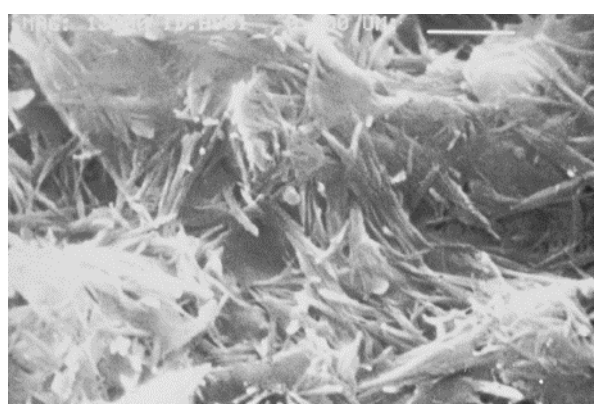

Figura 3. Attapulguita / Montmorillonita. Fuente: Oil Dri Corp. of America.

Las propiedades de la arcilla numeradas en la tabla 3 pueden ser ajustadas o modificadas durante su elaboración. La actividad de blanqueo se ve afectada por la selección de materia prima (Mineralogía) y el nivel de activación del adsorbente, incluyendo el nivel de acidez, la unidad libre, el tamaño y distribución de las partículas de absorbente pulverizadas. Las Condiciones recomendadas de blanqueo de aceite de palma se muestran en la tabla 4:

Tabla 4. Condiciones recomendadas de blanqueo de aceite de palma.

\begin{tabular}{|l|l|lr|}
\hline \multicolumn{4}{|c|}{ Condiciones recomendadas de blanqueo de aceite de palma } \\
\hline \multicolumn{1}{|c|}{ Condición } & Recomendación Teórica & \multicolumn{1}{|c|}{ Efecto Clave } \\
\hline Temperatura & $100-110{ }^{\circ} \mathrm{C}$ & $\begin{array}{l}\text { Viscosidad óptima del aceite para la } \\
\text { dispersión. Minimiza el aumento y } \\
\text { oxidación de los A.G.L. }\end{array}$ \\
\hline $\begin{array}{l}\text { Tiempo de } \\
\text { Contacto }\end{array}$ & $\begin{array}{l}\text { Proporciona interacción adecuada de } \\
\text { arcilla-aceite para la adsorción. }\end{array}$ \\
\hline Atmósfera & $20-50 \mathrm{~mm} \mathrm{Hg}$ & $\begin{array}{l}\text { Minimiza la Oxidación. Mejora la } \\
\text { Adsorción. }\end{array}$ \\
\hline Humedad & $0.2-0.3 \%$ & Mejora la Adsorción. \\
\hline
\end{tabular}

\section{Experimentación}

\section{Propiedades Iniciales del Aceite Crudo.}

Analizando las propiedades físicas evaluadas, se encuentra que el crudo 2 posee un alto valor de peróxidos ( $\mathrm{VP}=40)$, un alto nivel de porcentaje de FFA (5.2), y alto contenido de metales. El DOBI de 2.7 indica que esta muestra de aceite es de regular calidad. En pruebas posteriores se observa que estos niveles son reducidos considerablemente. Se deduce que este crudo es adecuado para realizar las presentes pruebas de estudio. Por experiencia los procesadores recomiendan iniciar el tratamiento con aceites de calidad superior $(\mathrm{DOBI}=3.5)$ para facilitar las operaciones de refinación.

Tabla 5. Datos experimentales característicos del aceite de palma crudo.

\begin{tabular}{|c|c|c|c|c|c|c|c|}
\hline & DOBI & VP & Cu & Fe & FFA & VA & P \\
\hline Crudo 1 & 2,8 & 1,6 & 0,1 & 0,9 & 2,2 & 10 & 36 \\
\hline Crudo 2 & 2,7 & 4.0 & 0,1 & 4,9 & 5.2 & 14 & 21 \\
\hline Crudo 3 & 2,5 & 2 & 0,1 & 3,6 & 2,2 & 13 & 19 \\
\hline Crudo 4 & 2 & 1,3 & 0,1 & 8,7 & 7,7 & 17 & 18 \\
\hline
\end{tabular}




\section{Tratamiento Ácido del Aceite Crudo de Palma}

La dosificación de $0.15 \%$ de ácido cítrico al $20 \%$ de concentración a $90^{\circ} \mathrm{C}$ con 60 minutos de tiempo de contacto, presento los mejores resultados en lo que se refiere a la disminución del contenido de metales. Esto plantea una inquietud para la realización de un estudio futuro enfocado en determinar las condiciones adecuadas en el desgomado.

\section{Determinación Preliminar de Arcilla y Dosis Adecuada.}

Teniendo en cuenta los comentarios y experiencia de los fabricantes, y analizando los valores obtenidos experimentalmente y aunque los valores de color y anisidina no fueron reducidos en forma dramática, como si sucedió al tratar el aceite crudo con las arcillas ácidoactivadas P4000 y Tonsil respectivamente. La arcilla B-80 neutra, controla y reduce eficazmente variables críticas como el valor de peróxido (VP), y FFA que son variables que al no ser controladas ataca y deterioran las propiedades del aceite de palma. Se considera esta arcilla como la más adecuada en lo que respecta al proceso de blanqueo de aceite de palma. La dosis que presente mejor resultado, aunque un poco alta fue del $2 \%$ en peso. Acorde con lo que se ejecuta en las plantas, se trata de una dosis normal, pero susceptible a ser disminuida. Como se menciono anteriormente la calidad inicial del aceite crudo esta también trascendental en aspectos relacionados con la dosificación.

\section{Valor Anisidina}

Los mejores resultados se presentaron con el tratamiento con arcilla tonsil, P4000 y B80 en forma descendiente. Vale la pena aclarar que el valor de anisidina expresa el contenido de hidrocarburos (aldehídos y cetona) generados como producto de oxidaciones secundarias de peróxidos y FFA. Se parte del hecho que al controlar los FFA y peróxidos, no se daría lugar a la formación de este tipo de hidrocarburos. La arcilla B80 no presento gran efectividad en la remoción de hidrocarburos, aún así se considera adecuada y es necesario analizar sus resultados frente a otras variables analizadas.

\section{Valor \%FFA}

El mejor resultado de la remoción de FFA lo presento la arcilla neutra B80 con dosis del $1 \%$. Este factor es clave en la determinación definitiva de la arcilla adecuada para el tratamiento del aceite de palma.

\section{Valor índice de Peróxidos}

Nuevamente el mejor resultado se obtuvo con la arcilla neutra B80, pero esta vez con dosis del $2 \%$. El porcentaje de remoción de peró0xido con estas condiciones fue superior a $60 \%$, con este resultado se perfila esta arcilla como la más adecuada en el blanqueo de aceite de palma.

\section{Valor del Color}

El color del aceite blanqueado no es indicador de la calidad de la arcilla utilizada, es un dato subjetivo y ejecutado por curiosidad del autor que por representatividad para el presente estudio. La arcilla más efectiva fue la número dos P4000 que redujo en más de $80 \%$ el color rojo inicial del aceite de palma. 


\section{Resultados}

Para la evaluación del blanqueo, se tuvo en cuenta el efecto de la temperatura y el efecto del tiempo de contacto.

\section{Incidencia del tiempo de contacto (Arcilla Aceite) en el Blanqueo.}

Al prolongar el tiempo de contacto hasta 30 minutos las variables de: coloración, color de saponificación, contenido de P-Fe decrecieron significativamente en comparación con otros tiempos de contacto. Según los resultados experimentales, el tiempo de contacto adecuado ha de ser de 30 minutos.

Tabla 6. Datos experimentales incidencia del tiempo de contacto entre la arcilla y el blanqueo.

\begin{tabular}{|c|c|c|c|c|c|}
\hline & Color & $\begin{array}{c}\text { Color de } \\
\text { Saponificación }\end{array}$ & P & Fe & Estabilidad \\
\hline Crudo & - & - & 21,4 & 4,9 & - \\
\hline Tratado & - & - & 5,3 & 1,9 & - \\
\hline $10 \mathrm{~min}$ & 3,5 & 6,7 & 3,8 & 1,2 & 16,4 \\
\hline $20 \mathrm{~min}$ & 3,4 & 6,6 & 2,3 & 0,9 & 16,6 \\
\hline $30 \mathrm{~min}$ & 3 & 6 & 1 & 0,6 & 21,3 \\
\hline $40 \mathrm{~min}$ & 3,1 & 6,4 & 1,2 & 0,7 & 18,3 \\
\hline
\end{tabular}

\section{Incidencia de la Temperatura en el Blanqueo}

$\mathrm{El}$ mejor resultado se obtuvo con temperatura de $140^{\circ} \mathrm{C}$ en todas las variables medidas. Sin embargo, se identifica que a temperatura de $110^{\circ} \mathrm{C}$, los valores de $\mathrm{P}-\mathrm{Fe}$, color son iguales a los medidos en la prueba de incidencia del tiempo de contacto. Lo que podría indicar que la temperatura adecuada estaría en el rango entre $110-140^{\circ} \mathrm{C}$. Adicionalmente los fabricantes manifiestan que no es aconsejable "blanquear" aceite de palma a altas temperaturas debido a que suele presentarse posterior al desodorizado un fenómeno de reversión de color el cual arruina cualquier proceso de refinamiento.

Tabla 7. Datos Experimentales Incidencia de la Temperatura en el Blanqueo.

\begin{tabular}{|c|c|c|c|c|c|}
\hline & Color & $\begin{array}{c}\text { Color de } \\
\text { Saponificación }\end{array}$ & $\mathbf{P}$ & Fe & Estabilidad \\
\hline Crudo & - & - & 21,4 & 4,9 & - \\
\hline Tratado & - & - & 5,3 & 1,9 & - \\
\hline $100^{\circ} \mathrm{C}$ & 3,8 & 7 & 3,2 & 0,7 & 13,5 \\
\hline $110^{\circ} \mathrm{C}$ & 3 & 6 & 1 & 0,67 & 21,3 \\
\hline $120^{\circ} \mathrm{C}$ & 2,8 & 5,9 & 0,9 & 0,2 & 22,9 \\
\hline $130^{\circ} \mathrm{C}$ & 2,7 & 5,9 & 0,4 & 0,08 & 23,2 \\
\hline $140^{\circ} \mathrm{C}$ & 2,5 & 5,5 & 0,4 & 0,07 & 22,2 \\
\hline
\end{tabular}

\section{Conclusiones}

$\square$ Aunque el aceite crudo de palma debido a su perfil lipídico debería poseer una excelente estabilidad oxidativa, en la práctica no la presenta debido a la actividad 
de sustancias que se encuentran presentes en él como componentes menores como son: La humedad, los A.G.L., las partículas metálicas, los fosfolípidos y los productos de oxidación.

$\square$ La calidad y la estabilidad de aceite de palma RBD, depende de la calidad de aceite crudo y de las condiciones a emplear en el proceso de refinado. Durante el desgomado se busca acondicionar partículas metálicas para que sean adsorbidas posteriormente en el blanqueo.

$\square$ La etapa más crítica del proceso de refinación de aceite de palma consiste en el cuidado del manejo del fruto de la palma. La calidad del aceite crudo determina en gran parte las medidas que necesita tomar el procesador a fin de cumplir con las especificaciones de calidad del aceite final. El blanqueo es el punto determinante para la eliminación de impurezas del aceite, y en la cual se determina la calidad final de los productos terminados.

■l blanqueo elimina ó disminuye los promotores y los productos de oxidación, es una operación importante en la refinación del aceite de palma; La calidad y estabilidad del aceite blanqueado depende tanto de la forma como se realizó la etapa de degomado, como la temperatura, y tiempo de contacto utilizados en el blanqueo.

$\square$ Las propiedades físicas del aceite tratado con la arcilla neutra B-80 presentaron mejores resultados frente a los obtenidos con las arcillas activas, específicamente los resultados de FFA, Valor de Peróxidos, los cuáles son considerados como promotores de oxidación. La arcilla neutra podría considerarse como la de mejor desempeño en la remoción de materiales pro-oxidantes.

El aceite de palma crudo de buena calidad (DOBI $=3.5$ ) generalmente produce productos finales de buena calidad, siempre y cuando las instalaciones y los controles de refinación tengan funcionamiento adecuado.

\section{Agradecimiento}

Los autores expresan sus agradecimientos a la Doctora Nelly Morales Pedraza, Coordinadora de la Especialización en Ingeniería de Procesos en Alimentos y Biomateriales de la UNAD, al Ingeniero Rodrigo Osorio, Jefe de Producción de Acegrasas S. A. y al Instituto Colombiano para el Desarrollo de la Ciencia y la Tecnología "Francisco José de Caldas - COLCIENCIAS, por su apoyo al desarrollo de la investigación en Colombia.

\section{Bibliografía}

Allendoerfer C., Fundamentos de Matemáticas Universitarias. Tercera Edición. Editorial McGraw Hill. Bogotá. 1981 Pág. 477.

[D] Andersen A., Refinación de Aceites y Grasas para usos Alimenticios. Editorial Montesó. Barcelona. 1956. Pág. 184 - 265. 
Dd Aguilar Miguel, El Libro de las Grasas. Editorial Alianza. 1993 Pág. 33, 54,55.

[D] Agudelo A. Recuperación de Tocoferoles en el Aceite Crudo de Palma. Tesis de Grado Ingeniería Química, Universidad América, Bogotá. 1998 Pág. 122.

[D] Bailey A., Aceites y Grasas Industriales. Editorial Reverté. Buenos Aires. 2da Edición. 1958. Pág. 4-9, 36-37, 510-517.

[C] Bayerfeing J.C., Caroteniodes as Colorants and Vitamina A Precursors. Technological and Nutritional Aplications, 1ra Edición. London Academic press N.Y. 1981. Pág. 938

[D] Bird R. B. Fenómenos de Transporte. 5ta Reimpresión. Editorial Reverté. México. 1998 Cap. 16.

[Dd Branan C., Soluciones Prácticas para el Ingeniero Químico. 2da Edición. Editorial McGraw Hill. Mexico. 2000. Pág. 210.

[L] Brooks D. Artículo : Bleaching Options for refining Palm Oil. AOCS 2000.

[D] Cengel Y., Termodinámica. 2da Edición. Editorial Mc Graw Hill. Mexico. 1996. Pág. 629.

[D] Dutton H.J., Determination of Fat Composition. Editorial Oils Chemist. 1968 Pág. $45-46$.

[D] FEDEPALMA. Anuario de la Palma, Editorial Oficina de Comunicación y Prensa de Fedepalma, 1ra Edición. Bogotá. 1998. Pág.120

[D] Geankopolis C. Procesos de Transporte y Operaciones Unitarias. Editorial CECSA México. 1998. Pág. 213-218.

[D] Gunstone F., An Introduction to the Chemistry and Biochemistry of the Fatty Acids and Their Glycerides. 2da Edición. Londres. Editorial Chapman and Hall. 1697. Pág. $132-145$.

[D] Hawley G. Diccionario de Química y de Productos Químicos. Barcelona. Editorial Omega 1985, Pág. 178.

[D] Hines A. Transferencia de Masa, fundamentos y aplicaciones. Editorial Prentice Hall. 1ra Edición, México. 1987. Pág. 568.

[C] Mc Cabe W, Operaciones Básicas en Ingeniería Química. Editorial Reverté. Barcelona. 1958 Vol 2. Pág. 901.

[D] Kent J., Manual de Riegel de Química Industrial. 1ra Edición. Editorial Continental. Mexico. 1984. Pág.384 385.

[D] Patterson H. W., Bleaching and Purifying Fats ans Oils. Editorial AOCS. Champaign. 1992. Pág.102 - 160.

[D] Producción, Análisis y Control de Calidad de Aceites y Grasas Comestibles. AMV Ediciones. 1998. Pág.141, 43,161, 258.

[D] Puig I., Curso General de Química. 12da Edición. Editorial Marin. 1961. Pág. 56

[C] Settle A. Handbook of Instrumental Techniques for Analitical Chemistry. Editorial Prentice Hall. 1ra Edición. USA. 1997. Pág. 436.

[Dd Silvestre A, D’andrea C. Toxicología de los alimentos. Editorial Hemisferio sur. 1ra Edición. Buenos Aires, 1995. Pág.415-427.

[C] Talen H., Recommended Methods for the Analysis of Drying Oils. Edotorial Butterworths. 1965. Pág. 165.

[D] Tamayo Héctor, Un Plan de Producción de la Palma de Aceite. Editorial Fedepalma.1978. Pág. 23.

[D] Tojo Gabriel, OCÓN Joaquín., Problemas en Ingeniería Química. Editorial Aguilar. Madrid . 3ra Edición .1968 Vol. 2, Pág. 324.

[D] Torres J. Análisis Numérico. ISBN: 958-651-479 Editorial UNAD. 2005, Bogotá. 
[D] Treybal E. Operaciones de Transferencia de Masa. Editorial Mc Graw Hill. 2da Edición. México.1998. Pág. 858.

[C] Velayuthon A. Procesamiento y Control de Aceite de Palma. Editorial Palmas. Vol. 18 No. 1. 1997. Pág. $19-33$.

[a] Weiss T., Foods and Their Uses. Editorial Wesport. Editorial Pub Co. 1.970 . Pág.69.

[Dd Zieller Steve., Food Fats and Oils. Editorial Acribia, 7ma Edición. 1996. Pág. 47 53. 